\title{
Desempenho de clones pré-imunizados de laranjeiras Valência e Natal no Norte do Estado de São Paulo
}

\author{
Eduardo Sanches Stuchi ${ }^{1,2 *}$, Eduardo Augusto Girardi ${ }^{1}$, Simone Rodrigues Silva ${ }^{3}$, \\ Otávio Ricardo Sempionato ${ }^{2}$, Luiz Gustavo Parolin², Gerd Walter Müller ${ }^{4} \&$ \\ Luiz Carlos Donadio ${ }^{5}$
}

\section{RESUMO}

As laranjas de maturação tardia são muito produzidas no Estado de São Paulo, principalmente para processamento de suco, dada sua elevada qualidade. As variedades Valência IAC e Natal IAC atendem a essa demanda, representando 38\% dos pomares em 2018. A extensão de safra nesse período do ano é relevante para fins do escalonamento da colheita e planejamento da indústria, desde que novas variedades apresentem parâmetros tecnológicos, como rendimento e concentração de sólidos solúveis, no mínimo similares aos padrões. Borbulhas de 14 clones de laranjeiras Valência e Natal foram coletados em campo na região de Bebedouro, sanitizados e pré-imunizados contra tristeza. Neste trabalho, seu desempenho foi avaliado sobre limoeiro Cravo em comparação às variedades de referência Valência IAC e Natal IAC em condições de sequeiro por 11 anos no Norte de São Paulo. O delineamento foi inteiramente casualizado com 16 tratamentos e seis repetições com uma árvore cada. O tamanho de planta e a produção de frutos foram muito similares entre os clones e variedades avaliados. Nenhum clone apresentou vantagens comparativas relevantes quanto a atributos de qualidade em relação às variedades de referência, embora diferenças entre tamanho de fruto, sólidos solúveis, rendimento de suco, acidez e ratio tenham sido observadas entre alguns clones. Natal IAC confirmou sua superioridade, mesmo em relação à Valência IAC, em função de seu maior índice tecnológico no Norte de São Paulo.

Termos de indexação: Citrus spp., melhoramento genético, produção, qualidade de frutos, variedades de copa.

\section{Performance of preimmunized clones of Valencia and Natal sweet oranges in the North of São Paulo state, Brazil}

\section{SUMMARY}

Late season oranges are widely grown in São Paulo, mainly for juice processing, because of their high quality. The varieties Valencia IAC and Natal IAC meet this demand, representing 38\% of the orchards in 2018. The extension of the harvest is important to stagger the crop to the industry,

\footnotetext{
${ }^{1}$ Embrapa Mandioca e Fruticultura, Cruz das Almas, BA, Brasil

${ }^{2}$ Estação Experimental de Citricultura de Bebedouro, Bebedouro, SP, Brasil

${ }^{3}$ Universidade de São Paulo - USP, Campus de Piracicaba, Piracicaba, SP, Brasil

${ }^{4}$ Instituto Agronômico, Campinas, SP, Brasil

${ }^{5}$ Universidade Estadual Paulista - UNESP, Campus de Jaboticabal, Jaboticabal, SP, Brasil

*Autor correspondente: Eduardo Sanches Stuchi, Estação Experimental de Citricultura de Bebedouro, Rodovia Faria Lima, Km 384, CEP 14713-000, Bebedouro, SP, Brasil. E-mail: eduardo.stuchi@embrapa.br.
} 
as long as new varieties present technological parameters such as juice yield and soluble solids at least similar to the standards. Fourteen clones of elite trees of Valencia and Natal were collected in the field in the region of Bebedouro city, and later sanitized and preimmunized against tristeza. In this work, their performance was evaluated on Rangpur lime in relation to the standard varieties, Valencia IAC and Natal IAC, under rain-fed conditions for eleven years in Northern São Paulo. Experimental design was completely randomized with 16 treatments, six replications and one tree in the plot. Tree size and fruit production were similar among the evaluated clones and varieties. None of the clones presented relevant advantages regarding fruit quality in relation to the standards, even though some differences were observed for fruit weight and size, soluble solids, acidity, juice yield and ratio between some clones. Natal IAC confirmed its superiority, even when compared with Valencia IAC, due to its higher technological index in Northern São Paulo.

Index terms: Citrus spp., breeding, yield, fruit quality, scion varieties.

\section{INTRODUÇÃO}

O Brasil é o maior produtor de laranjas [Citrus $\times$ sinensis (L.) Osbeck] e também o principal exportador de suco de laranja do mundo, seguido dos EUA. Nesses países, os Estados de São Paulo e da Flórida representam cerda de 80\% do total produzido para processamento (Neves et al., 2019), sendo que as variedades de laranja de maturação tardia são as mais cultivadas para processamento. No cinturão citrícola de São Paulo e Minas Gerais, as variedades tardias mais usadas são Valência e Natal, com participação de 27 e $11 \%$ do total de árvores (FUNDECITRUS, 2019), enquanto na Flórida a Valência representa aproximadamente 56\% dos pomares (USDA, 2017).

O predomínio dessas duas variedades se explica pela menor suscetibilidade ao vírus da tristeza dos citrus (CTV) do que a laranjeira Pera (Müller et al. 1999) e a maturação mais tardia de seus frutos. Além disso, apresentam desempenho agronômico e qualidade de suco excepcionais (Rouse, 2000; Pio et al., 2005; Girardi et al., 2017), notadamente para a fabricação de suco pasteurizado não concentrado (NFC, o mais valorizado pelos consumidores a partir dos anos 2000 (Neves et al., 2019). A Valência é a variedade de laranja mais plantada no mundo, sendo também conhecida como Valência Late, sendo de provável origem portuguesa (Saunt, 2000; Hodgson, 1967). Já a Natal é cultivada predominantemente no Brasil e trata-se de uma provável seleção de Valência obtida no país com maturação de frutos ligeiramente mais tardia do que a própria Valência (Castle \& Baldwin, 2011; Saunt, 2000; Donadio et al., 1995). A safra dessas variedades ocorre de setembro a dezembro em São Paulo (Pio et al., 2005).

A existência de diversas seleções e/ou clones regionais dessas variedades é bem conhecida, com variações em atributos horticulturais de interesse como tamanho de planta e de fruto, produtividade, época de maturação, qualidade e cor de suco, entre outras (Pardo et al. 2019;
Girardi et al., 2017; Castle \& Baldwin, 2011; Salibe, 2009; Tazima et al., 2008; Figueiredo et al., 2002; Hodgson, 1967). Estudaram-se clones velhos (old budlines) de Valência na Flórida com bom desempenho (Rouse, 2000). O trabalho de seleção é relevante para estender a safra dessas variedades, pois a maturação de frutos melhor distribuída durante a safra facilita o planejamento de colheita nas fazendas e de processamento nas indústrias, especialmente quando os parâmetros de qualidade são mais rigorosos como no caso do suco NFC.

Nos anos 1980 e 1990, selecionaram-se em campo plantas muito produtivas de clones velhos de Natal e Valência, aparentemente livres de sorose, exocorte e xiloporose, em Bebedouro-SP e municípios limítrofes. Esses clones apresentaram bom desempenho em Bebedouro quando enxertados em citrumelo Swingle $[C . \times$ paradisi Macfad. x Poncirus trifoliata (L.) Raf.] (Donadio et al., 1999). Plantas dos referidos clones velhos foram indexadas biologicamente e passaram por sanitização pela técnica de microenxertia de ápices caulinares in vitro (Roistacher et al., 1976; Navarro \& Juárez, 2007) e receberam pré-imunização contra o CTV com a estirpe PIAC dentro dos princípios da proteção cruzada (Costa \& Müller, 1980; Müller et al., 1999). Obtiveram-se, assim, novos clones microenxertados e pré-imunizados que foram disponibilizadas para estudos de campo. A pré-imunização contra o CTV é preconizada para todas as variedades de citros no programa de produção de material básico em São Paulo com vistas à prevenção da disseminação de isolados fortes (Carvalho et al., 2005).

Em continuidade aos trabalhos de seleção e sanitização mencionados, relatamos o desempenho dos clones pré-imunizados das laranjeiras Natal e Valência em comparação às variedades de referência Valência IAC e Natal IAC nas condições da região Norte do Estado de São Paulo. A extensão das diferenças de vigor, produção e qualidade de frutos entre as seleções são apresentadas, e as 
implicações de seu uso são discutidas frente à possibilidade de diversificação de variedades tardias que conjuguem suco de qualidade para NFC e maior escalonamento da colheita durante o pico da safra.

\section{MATERIAL E MÉTODOS}

O experimento foi instalado na Estação Experimental de Citricultura de Bebedouro, no Estado de São Paulo, Brasil (20 53' 16" S, 48 28' 11' 'W, $601 \mathrm{~m}$ de altitude). O clima da região é subtropical de montanha, com inverno moderado e seco e verão quente e chuvoso, sendo que a precipitação anual média foi de $1.560 \mathrm{~mm}$ e a temperatura média do ar de $26{ }^{\circ} \mathrm{C}$ durante o período de avaliação (Figura 1).

Compararam-se 14 clones pré-imunizados (Tabela 1), sendo nove de Natal e cinco de Valência, além das variedades de referência Valência IAC e Natal IAC, todos enxertados em limoeiro Cravo $(C . \times$ limonia Osbeck). O delineamento experimental foi inteiramente casualizado, com 16 tratamentos, seis repetições e uma planta na parcela, totalizando 96 plantas.

A implantação do experimento ocorreu em outubro de 1998, conduzido sem sistema de irrigação, com espaçamento de $7 \mathrm{~m}$ entre linhas de plantio x $4 \mathrm{~m}$ entre plantas. O solo é classificado como latossolo vermelho-escuro eutrófico de textura argilosa (38\% de argila), profundo e sem restrições químicas ou físicas $\left(\mathrm{pH}\left(\mathrm{CaCl}_{2}\right)=5,1 ; \mathrm{CTC}=56,3 \mathrm{mmol}_{\mathrm{c}} \cdot \mathrm{dm}^{-3}\right.$ a $\left.0-20 \mathrm{~cm}\right)$ (EMBRAPA, 1999). Os tratos culturais adotados foram os usualmente recomendados ao cultivo de laranja no Estado de São Paulo (Mattos Junior et al., 2005), porém não foram realizadas podas. As adubações seguiram as recomendações do Grupo paulista de adubação e calagem para citros - (GPACC, 1994), tendo sido ajustadas de modo que as doses acumuladas de nutrientes aplicadas por planta foram de $2317 \mathrm{~g}$ de $\mathrm{N}, 776 \mathrm{~g}$ de $\mathrm{P}_{2} \mathrm{O}_{5}$ e $1605 \mathrm{~g}$ de $\mathrm{K}_{2} \mathrm{O}$ do plantio até o ano de 2008.

A produção anual de frutos foi obtida por pesagem em balança digital e os resultados médios expressos em $\mathrm{kg}$ de frutos por planta nas safras de 2001 a 2008. Também foi calculado o índice de alternância de produção - IAP $(\mathrm{IAP}=1 / \mathrm{n}-1 \times\{|(\mathrm{a} 2-\mathrm{a} 1)| /(\mathrm{a} 2+\mathrm{a} 2)+|(\mathrm{a} 3-\mathrm{a} 2)| /(\mathrm{a} 3+\mathrm{a} 2)+\ldots+$ $|(\mathrm{a}(\mathrm{n})-\mathrm{a}(\mathrm{n}-1))| /(\mathrm{a}(\mathrm{n})+\mathrm{a}(\mathrm{n}-1))\})$, onde o IAP = índice de alternância de produção, $\mathrm{n}=$ número de safras avaliadas $\mathrm{e}$ a1, a2,..., a(n-1), a(n) = produção dos anos correspondentes (Pearce \& Dobersek-Urbanc, 1967). A precocidade de produção foi estimada pela divisão entre a produção acumulada nas quatro primeiras safras e a produção total no período.

No período 2006-2008, foram mensuradas anualmente as dimensões das plantas, sendo a altura (h) e diâmetro médio (dm) das copas obtidos com uma régua graduada, e posteriormente, calculou-se o volume de copa $\left(\mathrm{m}^{3}\right)$, com a fórmula utilizada

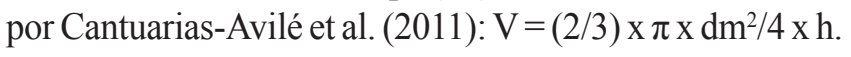

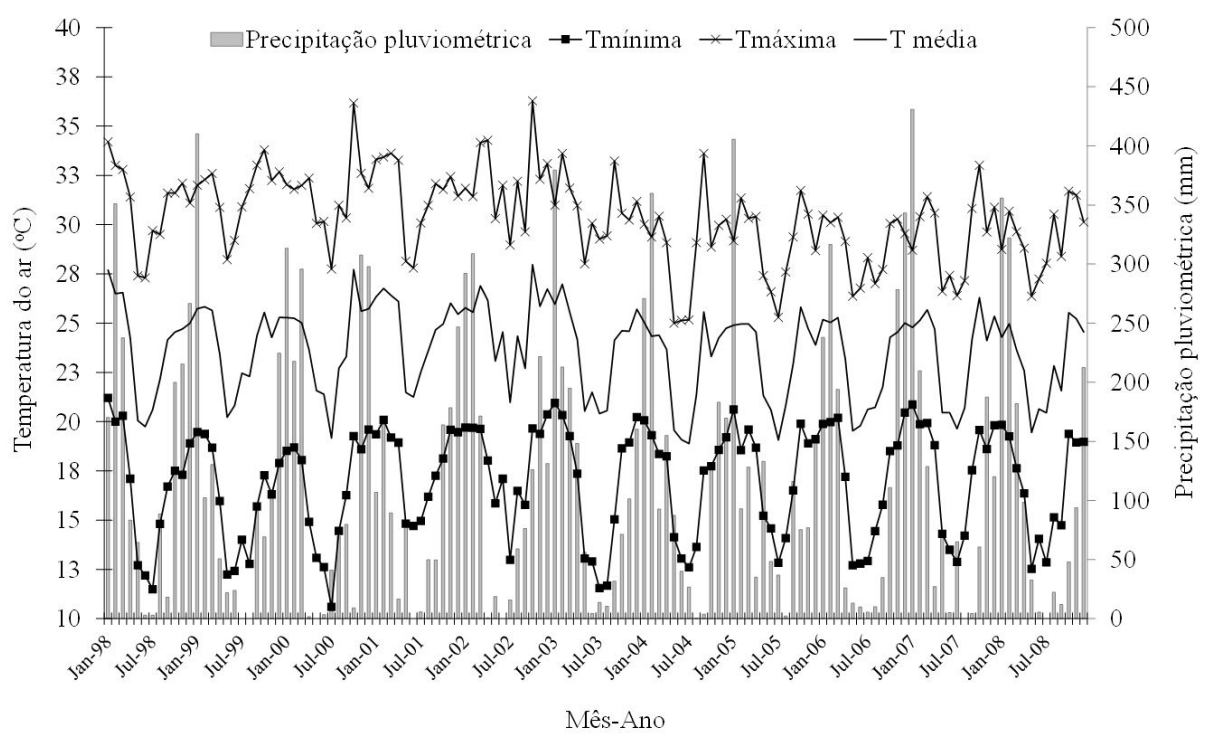

Figura 1. Médias mensais de temperatura do ar (máxima, mínima e média) e de precipitação pluviométrica na área experimental obtidas de estação meteorológica automatizada Campbell CR-10, no período 1998-2008 em Bebedouro, SP, Brasil 
Tabela 1. Designação experimental, origem e idade da planta-mãe na coleta de borbulhas para produção de 14 clones microenxertadas e pré-imunizados de laranjeiras Valência e Natal, selecionados em campo na região de Bebedouro, SP, Brasil

\begin{tabular}{|c|c|c|c|c|}
\hline \multirow{2}{*}{ Designação } & \multicolumn{3}{|c|}{ Origem do clone } & \multirow{2}{*}{$\begin{array}{r}\text { Idade } \\
\text { (anos) }\end{array}$} \\
\hline & Fazenda & Produtor & Município & \\
\hline Natal Vila São João-1948 & São João & Otto Mahle & Bebedouro & 40 \\
\hline Natal Pedro Sanches-1944 & n.d. & Pedro Sanches Filho & n.d. & 44 \\
\hline $\begin{array}{l}\text { Natal Milton Teixeira-Fazenda } \\
\text { Santa Terezinha }\end{array}$ & Santa Terezinha & Milton Teixeira & Bebedouro & n.d. \\
\hline Natal Felício Sasso- 1959 & Nova Aliança & Felício Sasso & Monte Azul Paulista & 29 \\
\hline Natal Ivan Aidar-1953 & Figueira & Ivan Aidar & Severínia & 35 \\
\hline Natal Fazenda União-35 anos & União & Francisco Medeiros Neto & Bebedouro & 35 \\
\hline Natal Nair Hernandes 1954 & Palmeiras & Nair Tasso Hernandes & Bebedouro & 34 \\
\hline Natal 2 & Santa Irene & Aracy Marques Caldeira & Bebedouro & n.d. \\
\hline Natal 1 & Santa Irene & Aracy Marques Caldeira & Bebedouro & n.d. \\
\hline Natal IAC & CCSM & $\mathrm{IAC}$ & Cordeirópolis & n.d. \\
\hline Valência 1 & Santa Irene & Aracy Marques Caldeira & Bebedouro & n.d. \\
\hline Valência 2 & Santa Irene & Aracy Marques Caldeira & Bebedouro & n.d. \\
\hline Valência 3 & Santa Irene & Aracy Marques Caldeira & Bebedouro & n.d. \\
\hline $\begin{array}{l}\text { Valência Jorge Ralston-Santa } \\
\text { Terezinha-1962 }\end{array}$ & Santa Terezinha & Jorge Uchoa Ralston & Bebedouro & 26 \\
\hline Valência Sérgio Pimenta-1944 & São José & Sérgio Pimenta & Olímpia & 44 \\
\hline Valência IAC & CCSM & IAC & Cordeirópolis & n.d. \\
\hline
\end{tabular}

CCSM = Centro de Citricultura Sylvio Moreira; IAC = Instituto Agronômico; n. d. = não definido.

Apenas as dimensões das plantas aos 10 anos são apresentadas. A eficiência produtiva média foi calculada pela divisão da produção de frutos por planta pelo volume da copa, expressos em $\mathrm{kg} \mathrm{m}^{-3}$, nos anos de 2006 a 2008.

A qualidade dos frutos foi avaliada nas safras de 2004 a 2008, exceto em 2007, com base em amostras de cinco frutos por parcela, coletadas sempre em outubro, época usual de maturação das variedades tardias na localidade. Avaliaram-se a massa, diâmetro e altura de frutos, concentração de sólidos solúveis (SS), medida em refratômetro (Palette PR-101, ATAGO, Tokyo, Japan), acidez total (AT), por titulação com hidróxido de sódio $(0,3125 \mathrm{~N})$, ratio calculado pela relação entre $\mathrm{SS} / \mathrm{AT}$, rendimento de suco (RS), usando uma extratora de ponto-de-venda (Otto 1800, OIC, Limeira, São Paulo, Brazil) e índice tecnológico $\left(\mathrm{kg} \mathrm{SS}\right.$ caixa $\left.{ }^{-1}\right)$, por IT $=[\mathrm{RS}$ x SS x 40,8 kg de laranja ou uma caixa padrão industrial] $\mathrm{x} 10.000^{-1}$.

Os dados obtidos foram submetidos à análise de variância e as médias comparadas pelo teste de Tukey $(p<0,05)$. As análises estatísticas foram realizadas com o auxílio do Software SISVAR 5.3. (Ferreira, 2011).

\section{RESULTADOS}

A produção média no período 2001-2008 foi equivalente para todos os clones avaliados, sendo em média de $60,8 \mathrm{~kg}$ planta $^{-1}$, o que correspondeu a 21,7 tha ${ }^{-1}$ em média. Quanto à precocidade de produção, também não ocorreram diferenças significativas entre os clones. Nas quatro primeiras safras avaliadas, foram produzidos, em média, $35 \%$ dos frutos do total acumulado do experimento. Na avaliação da alternância de safra, não houve diferença entre os clones, com IAP médio de 0,32 , sendo a alternância, de maneira geral, classificada como baixa, ou seja, com valore de IAP abaixo de 0,40 (Tabela 2).

Os clones Valência 3 e Natal Ivan Aidar apresentaram as menores alturas de planta e diâmetros da copa, porém os volumes de copa foram semelhantes aos das demais seleções no ano de 2008 (Tabela 2). Em média, as plantas alcançaram diâmetro, altura e volume de copa de 3,48 m, 2,81 m e 18,93 m³ , respectivamente. Contudo, em termos de eficiência produtiva média $\left(\mathrm{kg}\right.$ frutos $\mathrm{m}^{-3} \mathrm{de}$ copa), novamente não houve diferenças entre os clones pré-imunizados. 
Tabela 2. Produção média de frutos, índice de alternância (IAP) e precocidade de produção (PP) no período 2001-2008, eficiência produtiva média (EfP) no período 2006-2008 e dimensões das plantas em 2008 de 16 clones pré-imunizados das laranjeiras Natal e Valência, enxertadas sobre limoeiro Cravo, em Bebedouro, SP, Brasil

\begin{tabular}{|c|c|c|c|c|c|c|c|}
\hline \multirow{2}{*}{ Clone } & \multirow{2}{*}{$\frac{\text { Produção }}{\text { kg planta }^{-1}}$} & \multirow{2}{*}{ IAP* } & \multirow{2}{*}{$\begin{array}{c}\mathrm{PP} * * \\
\%\end{array}$} & \multirow{2}{*}{$\begin{array}{c}\mathrm{EfP} \\
\mathrm{kg} \mathrm{m}^{3}\end{array}$} & Diâmetro* & Altura & \multirow{2}{*}{$\frac{\text { Volume }}{\mathrm{m}^{3}}$} \\
\hline & & & & & \multicolumn{2}{|c|}{---------- $\mathrm{m}$--------- } & \\
\hline Natal Vila São João - 1948 & $67,4 \mathrm{a}$ & $0,26 \mathrm{a}$ & $36,3 \mathrm{a}$ & $6,20 \mathrm{a}$ & $3,63 \mathrm{a}$ & $2,86 \mathrm{a}$ & $20,93 \mathrm{a}$ \\
\hline Natal Pedro Sanches - 1944 & $61,0 \mathrm{a}$ & $0,31 \mathrm{a}$ & $37,0 \mathrm{a}$ & $6,00 \mathrm{a}$ & $3,63 \mathrm{a}$ & $2,86 \mathrm{a}$ & $20,22 \mathrm{a}$ \\
\hline Natal Milton Teixeira & $62,6 \mathrm{a}$ & $0,37 \mathrm{a}$ & $34,0 \mathrm{a}$ & $5,46 \mathrm{a}$ & $3,64 \mathrm{a}$ & $2,87 \mathrm{a}$ & $20,21 \mathrm{a}$ \\
\hline Natal Felício Sasso - 1959 & $59,7 \mathrm{a}$ & $0,33 \mathrm{a}$ & $40,7 \mathrm{a}$ & $5,17 \mathrm{a}$ & $3,72 \mathrm{a}$ & $3,09 \mathrm{a}$ & $22,42 \mathrm{a}$ \\
\hline Natal Ivan Aidar - 1953 & $64,7 \mathrm{a}$ & $0,35 \mathrm{a}$ & $39,8 \mathrm{a}$ & $4,70 \mathrm{a}$ & $3,16 \mathrm{bc}$ & $2,40 \mathrm{bc}$ & $18,12 \mathrm{a}$ \\
\hline Natal Fazenda União - 35 anos & $58,6 \mathrm{a}$ & $0,34 \mathrm{a}$ & $31,5 \mathrm{a}$ & $7,48 \mathrm{a}$ & $3,28 \mathrm{ab}$ & $2,77 \mathrm{ab}$ & $15,79 \mathrm{a}$ \\
\hline Natal Nair Hernandes & 66,0 a & $0,31 \mathrm{a}$ & $23,4 \mathrm{a}$ & $6,65 \mathrm{a}$ & $3,57 \mathrm{a}$ & $2,90 \mathrm{a}$ & $19,36 \mathrm{a}$ \\
\hline Natal 2 & $52,6 \mathrm{a}$ & $0,40 \mathrm{a}$ & $41,0 \mathrm{a}$ & $4,66 \mathrm{a}$ & $3,68 \mathrm{a}$ & $2,93 \mathrm{a}$ & 20,98 a \\
\hline Natal 1 & $60,8 \mathrm{a}$ & $0,26 \mathrm{a}$ & $35,5 \mathrm{a}$ & $6,02 \mathrm{a}$ & $3,40 \mathrm{ab}$ & $2,86 \mathrm{a}$ & $17,41 \mathrm{a}$ \\
\hline Natal IAC & $62,3 \mathrm{a}$ & $0,36 \mathrm{a}$ & $37,6 \mathrm{a}$ & $6,88 \mathrm{a}$ & $3,50 \mathrm{ab}$ & $2,75 \mathrm{abc}$ & $17,83 \mathrm{a}$ \\
\hline Valência 1 & $55,5 \mathrm{a}$ & $0,32 \mathrm{a}$ & $40,4 \mathrm{a}$ & 5,49 a & 3,63 a & $2,83 \mathrm{ab}$ & $19,79 \mathrm{a}$ \\
\hline Valência 2 & $63,7 \mathrm{a}$ & $0,35 \mathrm{a}$ & $33,3 \mathrm{a}$ & $7,20 \mathrm{a}$ & $3,53 \mathrm{ab}$ & $2,86 \mathrm{a}$ & $19,09 \mathrm{a}$ \\
\hline Valência 3 & $60,0 \mathrm{a}$ & $0,28 \mathrm{a}$ & $36,1 \mathrm{a}$ & $5,82 \mathrm{a}$ & $2,88 \mathrm{c}$ & $2,30 \mathrm{c}$ & $15,06 \mathrm{a}$ \\
\hline Valência Jorge Ralston - 1962 & $62,0 \mathrm{a}$ & $0,31 \mathrm{a}$ & $31,1 \mathrm{a}$ & $6,55 \mathrm{a}$ & $3,52 \mathrm{ab}$ & $2,81 \mathrm{ab}$ & $18,64 \mathrm{a}$ \\
\hline Valência Sérgio Pimenta - 1944 & 58,9 a & $0,33 \mathrm{a}$ & $32,7 \mathrm{a}$ & $6,46 \mathrm{a}$ & $3,39 \mathrm{ab}$ & $2,80 \mathrm{ab}$ & $17,32 \mathrm{a}$ \\
\hline Valência IAC & $63,0 \mathrm{a}$ & $0,28 \mathrm{a}$ & $37,1 \mathrm{a}$ & $4,93 \mathrm{a}$ & $3,48 \mathrm{ab}$ & $3,10 \mathrm{a}$ & $19,72 \mathrm{a}$ \\
\hline$C V(\%)$ & 29,2 & 3,72 & 28,9 & 26,7 & 3,06 & 7,56 & 25,50 \\
\hline
\end{tabular}

Médias seguidas da mesma letra minúscula na coluna não diferem entre si pelo teste de Tukey $(\mathrm{p}<0,05) ;{ }^{*}$ dados analisados com transformação por raiz quadrada de $\mathrm{x}+1$; **percentagem de produção nas quatro primeiras safras avaliadas; CV:coeficiente de variação.

Os resultados de qualidade de frutos no período 2004-2008 podem ser observados na Tabela 3. Destaca-se Valência IAC, com os maiores frutos em geral, pesando em média $223 \mathrm{~g}$, altura e diâmetro de fruto de 7,73 e 7,41 cm. No outro extremo, clones com as menores massa dos frutos foram Natal Vila São João - 1948, Natal Pedro Sanches - 1944, Natal Fazenda União - 35 anos, Natal Nair Hernandes, Natal 2, Natal 1, Natal IAC, Valência 3 e Valência Jorge Ralston - 1962, enquanto as outras laranjeiras apresentaram médias intermediárias. Resultados semelhantes ocorreram para altura e diâmetro de frutos.

Natal Vila São João - 1948 apresentou maior percentual de suco $(52,11 \%)$ em relação a Valência IAC $(47,22 \%)$ e Natal $2(46,34 \%)$, que por sua vez também foi inferior a outros cinco clones, incluindo a variedade de referência, Natal IAC (51,30\%). Em outubro, no período 2004-2008, a laranjeira Natal IAC apresentou SS (11,55 ${ }^{\circ}$ Brix) superior ao de Natal Milton Teixeira, Natal Fazenda União - 35 anos, Valência 2 e Valência Jorge Ralston - 1962 (média de 9,40 ${ }^{\circ}$ Brix), sem diferir dos demais clones que apresentaram de 10,0 a 10,7 ${ }^{\circ}$ Brix.
Para acidez do suco, apenas os frutos de Natal Vila São João - 1948 e Natal IAC, com quase 1,0\% de acidez, diferiram dos de Valência 2 (0,68\%) (Tabela 3).

Quanto à classificação das variedades pelo ratio do suco, no mês de outubro, Valência $2(14,01)$ foi superior a Natal 1, Natal Fazenda União - 35 anos, Natal Vila São João - 1948, Natal Pedro Sanches - 1944, Valência 1, Valência Jorge Ralston - 1962 e Valência Sérgio Pimenta - 1944, todas abaixo de 11,82, porém sem que todas essas diferissem de Valência IAC $(12,77)$, Natal IAC $(12,14)$ e demais clones. A laranjeira Natal IAC resultou, por fim, em maior quantidade de sólidos solúveis por caixa de fruto, 2,42 $\mathrm{kg}$ de SS caixa $^{-1}$, em relação a Natal Milton Teixeira, Natal Felício Sasso - 1959, Natal 2, Natal Fazenda União - 35 anos, Valência 2, Valência Jorge Ralston - 1962 e mesmo Valência IAC, todas rendendo abaixo de $2,02 \mathrm{~kg}$ de $\mathrm{SS}$ caixa $^{-1}$, sem diferir dos demais clones (Tabela 3 ).

\section{DISCUSSÃO}


Tabela 3. Análise tecnológica de frutos de 16 clones pré-imunizados das laranjeiras Natal e Valência, enxertadas sobre limoeiro Cravo, médias no período 2004-2008*, exceto o ano de 2007. Bebedouro, SP, Brasil

\begin{tabular}{|c|c|c|c|c|c|c|c|c|}
\hline \multirow{2}{*}{ Clone } & Massa & Altura & Diâmetro & Suco & Sólidos & Acidez & \multirow{2}{*}{ ratio } & \multirow{2}{*}{$\frac{\mathrm{IT}}{\mathrm{kg} \mathrm{SS} * *}$} \\
\hline & $\mathrm{g}$ & \multicolumn{2}{|c|}{--------- cm -------- } & $\%$ & Brix & $\%$ & & \\
\hline Natal Vila São João - 1948 & $171 \mathrm{c}$ & $7,06 \mathrm{bc}$ & $6,76 \mathrm{c}$ & $52,11 \mathrm{a}$ & $10,68 \mathrm{ab}$ & $0,96 \mathrm{a}$ & $11,34 \mathrm{~b}$ & $2,27 \mathrm{ab}$ \\
\hline Natal Pedro Sanches - 1944 & $180 \mathrm{cb}$ & $7,04 \mathrm{bc}$ & $6,86 \mathrm{bc}$ & $51,71 \mathrm{ab}$ & $10,12 \mathrm{ab}$ & $0,89 \mathrm{ab}$ & $11,52 \mathrm{~b}$ & $2,14 a b$ \\
\hline Natal Milton Teixeira & $205 \mathrm{ab}$ & 7,38 abc & $7,27 \mathrm{ab}$ & $48,50 \mathrm{abc}$ & $9,58 \mathrm{~b}$ & $0,77 \mathrm{ab}$ & $13,10 \mathrm{ab}$ & $1,91 \mathrm{~b}$ \\
\hline Natal Felício Sasso - 1959 & $198 \mathrm{abc}$ & $7,41 \mathrm{abc}$ & $7,11 \mathrm{abc}$ & $48,79 \mathrm{abc}$ & $9,95 \mathrm{ab}$ & $0,78 \mathrm{ab}$ & $12,94 \mathrm{ab}$ & $1,98 \mathrm{~b}$ \\
\hline Natal Ivan Aidar - 1953 & $195 \mathrm{abc}$ & $7,47 \mathrm{ab}$ & $7,03 \mathrm{abc}$ & $49,92 \mathrm{abc}$ & $10,09 \mathrm{ab}$ & $0,86 \mathrm{ab}$ & $11,92 \mathrm{ab}$ & $2,05 a b$ \\
\hline $\begin{array}{l}\text { Natal Fazenda União - } 35 \\
\text { anos }\end{array}$ & $182 \mathrm{cb}$ & $7,06 \mathrm{bc}$ & $6,91 \mathrm{bc}$ & $51,42 \mathrm{ab}$ & $9,29 \mathrm{~b}$ & $0,86 \mathrm{ab}$ & $11,23 \mathrm{~b}$ & $1,95 \mathrm{~b}$ \\
\hline Natal Nair Hernandes & $179 \mathrm{cb}$ & $7,08 \mathrm{bc}$ & $6,84 \mathrm{bc}$ & $50,69 a b c$ & $10,27 \mathrm{ab}$ & $0,86 \mathrm{ab}$ & $12,29 \mathrm{ab}$ & $2,12 a b$ \\
\hline Natal 2 & $173 \mathrm{cb}$ & $6,93 \mathrm{c}$ & $6,89 \mathrm{bc}$ & $46,34 \mathrm{c}$ & $10,10 \mathrm{ab}$ & $0,77 \mathrm{ab}$ & $13,23 \mathrm{ab}$ & $1,92 \mathrm{~b}$ \\
\hline Natal 1 & $185 \mathrm{cb}$ & $7,22 \mathrm{bc}$ & $6,94 \mathrm{bc}$ & $51,10 \mathrm{ab}$ & $9,99 \mathrm{ab}$ & $0,87 \mathrm{ab}$ & $11,74 \mathrm{~b}$ & $2,09 a b$ \\
\hline Natal IAC & $182 \mathrm{cb}$ & $7,13 \mathrm{bc}$ & $6,87 \mathrm{bc}$ & $51,30 \mathrm{ab}$ & $11,55 \mathrm{a}$ & $0,98 \mathrm{a}$ & $12,14 \mathrm{ab}$ & $2,42 \mathrm{a}$ \\
\hline Valência 1 & $203 \mathrm{abc}$ & 7,27 abc & $7,19 \mathrm{abc}$ & $47,78 \mathrm{abc}$ & $10,17 \mathrm{ab}$ & $0,88 \mathrm{ab}$ & $11,77 \mathrm{~b}$ & $2,00 a b$ \\
\hline Valência 2 & $191 \mathrm{abc}$ & $7,31 \mathrm{abc}$ & $7,03 \mathrm{abc}$ & $48,85 \mathrm{abc}$ & $9,31 \mathrm{~b}$ & $0,68 \mathrm{~b}$ & $14,01 \mathrm{a}$ & $1,86 \mathrm{~b}$ \\
\hline Valência 3 & $183 \mathrm{cb}$ & $7,10 \mathrm{bc}$ & $6,86 \mathrm{bc}$ & $49,97 \mathrm{abc}$ & $10,43 \mathrm{ab}$ & $0,81 \mathrm{ab}$ & $13,10 \mathrm{ab}$ & $2,12 a b$ \\
\hline $\begin{array}{l}\text { Valência Jorge Ralston } \\
-1962\end{array}$ & $184 \mathrm{cb}$ & $7,19 \mathrm{bc}$ & $6,97 \mathrm{abc}$ & $50,94 \mathrm{abc}$ & $9,44 \mathrm{~b}$ & $0,81 \mathrm{ab}$ & $11,82 \mathrm{~b}$ & $1,96 \mathrm{~b}$ \\
\hline $\begin{array}{l}\text { Valência Sérgio Pimenta } \\
-1944\end{array}$ & $195 \mathrm{abc}$ & 7,29 abc & $7,03 \mathrm{abc}$ & $51,69 \mathrm{ab}$ & $9,80 \mathrm{ab}$ & $0,86 \mathrm{ab}$ & $11,55 \mathrm{~b}$ & $2,07 \mathrm{ab}$ \\
\hline Valência IAC & $223 \mathrm{a}$ & 7,73 a & $7,41 \mathrm{a}$ & $47,22 \mathrm{bc}$ & $10,33 \mathrm{ab}$ & $0,83 \mathrm{ab}$ & $12,77 \mathrm{ab}$ & $2,02 \mathrm{~b}$ \\
\hline$C V(\%)$ & 8,38 & 3,35 & 3,09 & 4,45 & 8,00 & 12,87 & 8,37 & 10,01 \\
\hline
\end{tabular}

Médias seguidas da mesma letra minúscula na coluna não diferem entre si pelo teste de Tukey $(p<0,05)$; *Frutos coletados no mês de outubro; ${ }^{* *} \mathrm{~kg}$ de sólidos solúveis por caixa de 40,8 kg; IAC = Instituto Agronômico; CV = coeficiente de variação IT = Índice tecnológico; SS = Sólidos solúveis.

Neste trabalho, as diferenças de características biométricas, de produção e de qualidade de frutos foram discretas entre as variedades tardias de referência em São Paulo, Valência IAC e Natal IAC, e diversos clones coletados a campo e posteriormente pré-imunizados. O senso comum é que há grande similaridade entre as características das diferentes seleções de Valência e mesmo entre a Natal e a Valência (Hodgson, 1967; Donadio et al., 1995; Saunt, 2000; Pio et al., 2005; Ollitraut \& Navarro, 2012). Considerando-se que o experimento foi conduzido sem uso de irrigação em região sujeita à seca e que a densidade de plantio foi de 357 plantas ha ${ }^{-1}$, a produtividade média estimada para o conjunto de variedades estudadas no período 2001-2008 foi de 21,84 t ha ${ }^{-1}$. Esse valor é praticamente a metade da média de produtividade paulista em pomares comerciais bem mais adensados em 2019 (Fundo de Defesa da Citricultura, 2019). Contudo, no experimento a produção média por plantas foi de $61,17 \mathrm{~kg}$, próxima a valores médios em safras recentes, indicando que a produtividade poderá ser ampliada em plantios empregando espaçamento e manejo ajustados.

Na Florida, não houve diferença prática entre 18 variedades tardias estudadas, mas se destacaram a maturação muito tardia da laranja Natal e a boa produção de frutos com alta qualidade de Appleby, que é um clone de Valência proveniente da Austrália. No entanto, no mesmo trabalho, as variedades de referência foram as que produziram mais: Valência 10-12-7 "old budline clone" e Valência 9-8-17 "nucelar old line", indicando que clones velhos podem se equiparar a clones novos (Castle \& Baldwin, 2011), como relatado aqui. Ressalta-se que no presente estudo avaliaram-se clones velhos que haviam passado por microenxertia e pré-imunização e, dessa forma, podem ter sido revigorados durante o processo. Diferenças na produtividade entre seleções nucelares de Valência introduzidas de outros países foram reportadas tanto em São Paulo como no Paraná (Girardi et al., 2017; Tazima et al., 2008), ao contrário do observado para as seleções microenxertadas no presente trabalho. 
As variedades Natal IAC e Valência IAC são de origem nucelar e, devido a isso, era esperado que fossem mais vigorosas, uma vez que se sabe que clones nucelares são usualmente mais robustos e de crescimento mais rápido do que clones velhos (Cameron \& Soost, 1982; Salibe, 2017). No entanto, as diferenças de tamanho de planta e de eficiência de produção apresentadas neste estudo estão dentro de uma faixa de pouco efeito prático, embora em outro estudo na região tenham se observado diferenças nessas mesmas variáveis para outras seleções de Valência e Natal (Girardi et al., 2017). Partes dessas seleçõesforam introduzidas de outros países por sementes, enquanto as seleções locais foram obtidas de plantas de campo, assumidas como sendo de clone velho das laranjeiras Natal e Valência comuns, vindo daí o comportamento distinto nos dois estudos, provavelmente. O porta-enxerto de limoeiro Cravo utilizado neste trabalho sabidamente induz vigor elevado às copas em geral, notadamente em cultivo de sequeiro, o que também pode ter mascarado diferenças de vigor inerentes aos clones estudados.

As principais variações apresentadas entre as seleções pré-imunizadas se devem a atributos de qualidade dos frutos, o que é relevante, pois permite identificar clones com maior adequação ao processamento de suco NFC ou mesmo com diferentes épocas de maturação e tamanho de fruto visando mercado in natura. A massa média dos frutos (189 g) dos clones foi superior aos relatados por Nonino (1995), 156 g para Natal e 165 g para Valência, e ligeiramente superior e inferior ao encontrado para Valências em Londrina e Nova Esperança, Paraná, 175 g e 193 g, respectivamente (Auler et al., 2009; Tazima et al., 2008). Apenas seis clones produziram frutos dentro da chamada faixa A da cotação do CEAGESP: Natal Milton Teixeira, Natal Felício Sasso - 1959, Natal Ivan Aidar - 1953, Valência 1, Valência 2, Valência Sérgio Pimenta-1944 e Valência IAC, ou seja, frutos com diâmetro equatorial maior que $70 \mathrm{~mm}$, são os que atingem os melhores preços (CEAGESP, 2017).

Para Natal IAC, embora tenha obtido maior valor de sólidos solúveis, este foi menor que o encontrado por Girardi et al. (2017) (12,9 a 13,1 $\left.{ }^{\circ} \mathrm{Brix}\right)$, possivelmente devido ao porta-enxerto de limoeiro Cravo em comparação a tangerineira Sunki (C. sunki Hort. ex Tanaka) e citrumelo Swingle [C. paradisi Macfad. x Poncirus trifoliata (L.) Raf.] estudados por esses autores. Na Flórida, o porta-enxerto de limoeiro Rugoso Vangasay (C. jambhiri Lush) induziu o menor valor de SS, $11,1^{\circ}$ Brix na média de seis seleções de Valência estudadas, enquanto o maior valor foi de $13,3^{\circ}$ Brix com média de $12,1^{\circ}$ Brix para 16 variedades de porta-enxertos (Rouse, 2000). Por outro lado, Nonino (1995) relatou que o teor de SS para Natal se situa entre 10,56 e $11,83^{\circ}$ Brix, faixa em que se enquadraram a Natal IAC e Natal Vila São João. A única seleção que atingiu SS considerado como ideal para produção de NFC foi a Natal IAC $\left(11.55^{\circ}\right.$ Brix), de acordo com Darros-Barbosa \& Curtolo (2005). Entretanto segundo os mesmos autores, a produção industrial de suco ocorre com suco a partir de $10{ }^{\circ}$ Brix, valor que não foi apresentado pelas seleções Natal Milton Teixeira, Natal Felício Sasso - 1959, Natal Fazenda União - 35 anos, Natal 1, Valência 2, Valência Jorge Ralston - 1962 e Valência Sérgio Pimenta - 1944.

Diferença nos valores de IT ( $\mathrm{kg}$ SS por caixa de $40,8 \mathrm{~kg}$ ) entre seleções de Valência foram encontradas no Norte do Estado do Paraná, onde Valência e Valência 718 apresentaram valores semelhantes, 2,2 $\mathrm{kg} \mathrm{SS}$ caixa $^{-1}$, superiores a Valência Late 1138, 1,95 kg SS caixa ${ }^{-1}$ (Tazima et al., 2008). Neste estudo, apenas Natal IAC e Natal Vila São João apresentaram valores semelhantes aos relatados pelos autores supracitados. Em outro estudo na mesma região, o valor de IT para Valência 718, acesso I-94, enxertada em limão Cravo foi de 2,16 kg SS (Auler et al., 2009). Variedades de origem nucelar e outras obtidas de clones velhos, mas, que haviam passado por microenxertia, apresentaram faixa de índice tecnológico similar nos EUA (Rouse, 2000), fato que também se observou no presente trabalho.

A Natal é considerada a mais tardia das cultivares de maior expressão comercial, mas Folha Murcha e Charmute de Brotas têm um período de colheita ainda mais extenso (Donadio et al., 1995; Saunt, 2000; Castle \& Baldwin, 2011; Carvalho et al., 2019). Entretanto, alguns clones de Natal estudados nesse trabalho foram menos tardios, considerando-se que a colheita de frutos foi realizada sempre em outubro, a exemplo de Felício Sasso, Milton Teixeira e Natal 2 com ratio médio de 13,09 em relação à média de 11,74 para demais clones de Natal. Por outro lado, alguns clones de Valência foram tão tardios quanto a maioria dos clones de Natal: Sérgio Pimenta-1944, Valência 1 e Jorge Ralston-Santa Terezinha-1962, com ratio médio de 11,71, enquanto os clones de Valência 2, 3 e IAC apresentaram uma média de 13,29. Diferenças de período de maturação entre variedades e seleções de Valência foram reportadas consistentemente na África do Sul, onde 13 variedades atendem um período que vai de meados de maio até meados de agosto considerando-se um ratio 10:1 (Lee,2019.). 
Na Flórida, Valência Frost mostrou frutos com ratio superior a outras 12 variedades quando enxertada em citrumelo Swingle tanto aos quatro como aos oito anos de idade (Castle \& Baldwin, 2011). Ainda, seleções de Valência com maturação antecipada de duas a três semanas em relação à Valência padrão, como Delta Seedless e Midknight, são conhecidas há bastante tempo (Hodgson, 1967) e têm crescido em termos de superfície cultivada (Pardo et al. 2019). Contrariamente, três seleções - Valência, Valência 718 e Valência Late 1138 - apresentaram valores de ratio semelhantes aos do corrente trabalho em Londrina-PR na média de 10 anos (Tazima et al., 2008). Essas variações de época de maturação, mesmo que correspondam a poucas semanas, são relevantes do ponto de vista comercial e operacional, pois permitem escalonar melhor a colheita durante o pico da safra das tardias.

Devido à carência de informações cientificas sobre seleções mais tardias de Valência, Sérgio Pimenta-1944, Valência 1 e Jorge Ralston-Santa Terezinha-1962 merecem atenção, pois, além de ratio menor no mês de outubro, apresentaram atributos de qualidade próximos aos de Valência IAC. A Valência 2, seguida pela Valencia 3, se mostraram como as mais precoces. Já entre os clones de Natal, Felício Sasso, Milton Teixeira e Natal 2 se mostraram como os mais precoces em relação a Natal IAC, Natal 1, Fazenda União, Vila São João e Pedro Sanches. Novos estudos podem evidenciar valores de rendimento em suco e SS mais adequados destes clones, desde que sejam enxertados em porta-enxertos mais adequados e/ ou em outras condições edafoclimáticas e de manejo. Donadio et al. (1999) relataram que as seleções de Valência e Natal acima mencionadas se prestam adequadamente ao processamento de suco concentrado.

Em resumo, Natal IAC e Valência IAC se mantêm superiores ou com as melhores características tecnológicas dos frutos em relação aos demais clones estudados neste experimento. Na Flórida, estudos com 18 seleções tardias, incluindo oito clones de Valência, concluíram que nenhuma dessas seleções se destacou frente aos dois clones de Valência mais populares e cultivados comercialmente naquele estado, 10-12-7 e 9-8-17 (Castle $\&$ Baldwin, 2011). O desempenho ligeiramente superior da Natal IAC observado no presente estudo espelha a sua participação no quadro varietal atual do cinturão citrícola, em que os pomares de Natal representam $11 \%$ do total (FUNDECITRUS, 2019), bem como sua adequação diferenciada para processamento de suco pasteurizado em função de alto SS e maior índice tecnológico.

\section{AGRADECIMENTOS}

À Fundação de Amparo à Pesquisa do Estado de São Paulo (FAPESP) pelo auxílio financeiro (Processos 1995/09283-5 e 2004/16077-3), ao Conselho Nacional de Desenvolvimento Científico e Tecnológico (CNPq) pela bolsa de produtividade do primeiro autor e à Fundação Coopercitrus Credicitrus (FCC) pela cessão área experimental, suporte técnico e administrativo.

\section{REFERÊNCIAS}

Auler, P. A. M., Fiori-Tutida, A. C. G., \& Scholz, M. B. S. (2009). Qualidade industrial e maturação de frutos de laranjeira Valência sobre seis porta-enxertos. Revista Brasileira de Fruticultura, 31(4), 1158-1167.

Cameron, J., \& Soost, R. (1982). Breeding and development. California Agriculture, 36(11), 4-6.

Cantuarias-Avilé, S., Mourão, T., Filho, F. A. A., Stuchi, E. S., Silva, S. R., \& Espinoza-Nuñes, E. (2011). Horticultural perfomance of 'Folha Murcha' sweet orange onto twelve rootstocks. Scientia Horticulturae, 129(2), 259-265.

Carvalho, S. A., Graf, C. C. D., \& Violante, A. R. (2005). Produção de material básico e propagação. In D. Mattos Junior, J. D. De Negri, R. M. Pio, \& J. Pompeu Junior (Eds). Citros (pp. 279-316). Campinas: Instituto Agronômico e Fundag.

Carvalho, S.A, Girardi, E.A., Mourão Filho, F.A.A, Ferrarezi, R.S. \& Coletta Filho, H. D.. (2019). Advances in citrus propagation in Brazil. Revista Brasileira de Fruticultura, 41(6), e-422. http://dx.doi.org/10.1590/0100-29452019422.

Castle, W. S., \& Baldwin, J. C. (2011). Young-tree performance of juvenile sweet orange scions on Swingle citrumelo rootstock. HortScience, 46(4), 541-552.

Companhia de Entrepostos e Armazéns Gerais de São Paulo - CEAGESP. (2017). Cartilha técnica: a medida das frutas (16 p.). São Paulo: CEAGESP.

Costa, A. S., \& Müller, G. W. (1980). Tristeza controlled by cross protection, a US-Brazil cooperative success. Plant Disease, 64, 538-541.

Darros-Barbosa, R., \& Curtolo, J. E. (2005). Produção industrial de suco e subprodutos cítricos. In D. Mattos Junior, J. D. De Negri, R. M. Pio, \& J. Pompeu Junior 
(Eds.), Citros (pp. 839-870). Campinas: Instituto Agronômico e Fundag.

Donadio, L. C., Figueiredo, J. O., \& Pio, R. M. (1995). Variedades citricas brasileiras (228 p.). Jaboticabal: FUNEP.

Donadio, L. C., Stuchi, E. S., Pozzan, M., \& Sempionato, O. R. (1999). Novas variedades de laranja para indústria (42 p.). Jaboticabal: FUNEP.

Empresa Brasileira de Pesquisa Agropecuária-EMBRAPA. (1999). Sistema brasileiro de classificação de solos (412 p.). Brasília: Embrapa Produção de Informação; Rio de Janeiro: Embrapa Solos.

Ferreira, D. F. (2011). SISVAR: A computer statical analysis system. Ciência e Agrotecnologia, 35(6), 1039-1042.

Figueiredo, J. O., Laranjeira, F. F., \& De Negri, J. D. (2002). Comportamento de 42 variedades de laranja doce enxertadas em dois porta-enxertos em área de alta incidência de clorose variegada dos citros. In Anais do $17^{\circ}$ Congresso Brasileiro de Fruticultura. Belém: Sociedade Brasileira de Fruticultura.

Fundo de Defesa da Citricultura-FUNDECITRUS. (2019). Inventário de árvores e estimativa da safra de laranja 2019/20 do cinturão citrícola de São Paulo e Triângulo/ Sudoeste mineiro. Araraquara: FUNDECITRUS. Recuperado em 8 de janeiro de 2019, de https://www.fundecitrus.com. br/pdf/pes_relatorios/2019_05_24_Invent $\%$ C $3 \% A 1$ rio_e Estimativa_do_Cinturao_Citricola_2019-2020.pdf

Girardi, E. A., Cerqueira, T. S. C., Cantuarias-Avilés, T. E., Silva, S. R., \& Stuchi, E. S. (2017). Sunki mandarin and Swingle citrumelo as rootstocks for rain-fed cultivation of late-season sweet orange selections in northern São Paulo state, Brazil. Bragantia, 76(4), 501-511.

Grupo Paulista de Adubação e Calagem para Citros - GPACC. (1994). Recomendações para adubação e calagem para citros no Estado de São Paulo (3. ed., 27 p). Cordeirópolis: GPACC.

Hodgson, R. W. (1967). Horticultural varieties of citrus. In W. Reuther, L. D. Batchelor \& H. J. Webber (Eds.), The citrus industry (Vol. 1, pp. 431-591). Riverside: University of California.

Lee, A. Citrus varieties. In: Citrus Research International - CRI. Module 3. Citrus varieties (Citrus Postharvest Series Learner Guide). South Africa: Citrus Research International (CRI). Recuperado em 11 de novembro de 2019, de https://www.citrusresourcewarehouse.org. za/home/document-home/learning-aids-and-resources/ ca-citrus-av-series-learning-material/citrus-post-harvestseries/1125-cphs-learning-material-module-03-citrus-varieties/ file

Mattos Junior, D., De Negri, J. D., Figueiredo, J. O., \& Pompeu Junior, J. (2005). Citros: principais informações e recomendações de cultivo. Campinas: Instituto Agronômico e Fundag. Recuperado em 16 de fevereiro de 2018, de http:// www.iac.sp.gov.br/imagem_informacoestecnologicas/43. pdf. Acessado em: 16 fev. 2018.

Müller, G. W., Targon, M. L. P. N., \& Machado, M. A. (1999). Trinta anos de uso do clone pré-imunizado Pêra IAC na citricultura paulista. Laranja, 20, 399-408.

Navarro, L., \& Juárez, J. (2007). Shoo-tip grafting in vitro: impact in the citrus Industry and research applications. In I. A. Khan (Eds.), Citrus genetics, breeding and biotechnology (pp. 353-364.) Wallingford: CABI.

Neves, M. F., Trombin, V. G., Mônaco Neto, L. C., \& Kalaki, R. B. (2019). Orange juice chain: past, present and future (174 p.). Western Cape, South Africa: Quickfox Publishing; Citrus Growers Association.

Nonino, E. A. (1995). Variedades de laranjas para fabricação de sucos. Laranja, 16(1), 119-132.

Ollitraut, P., \& Navarro, L. (2012). Citrus. In M. L. Badenes \& D. H. Byrne (Eds.), Fruit breeding: handbook of plant breeding (Vol. 8, 623 p.). New York: Springer.

Pardo, J., Soler, G., \& Buj, A. (2019). Variedades comerciales de citricos - fichas varietales. Recuperado em 28 de maio de 2019, de http://www.ivia.gva.es/va/ variedades

Pearce, S. C., \& Dobersek-Urbanc, S. (1967). The measurements of irregularity in growth and cropping. Journal of Horticultural Science, 42, 295-305.

Pio, R. M., Figueiredo, J. O., Stuchi, E. S., \& Cardoso, S. A. B. (2005). Variedades copas. In D. Mattos Junior, J. D. De Negri, R. M. Pio, \& J. Pompeu Junior (Eds.), Citros (pp 39-60). Campinas: Instituto Agronômico e Fundag.

Roistacher, C. N., Navarro, L., \& Murashige, T. (1976). Recovery of citrus selections free of several viruses, exocortis viroid, and Spiroplasma citri by shoot tip grafting in vitro. In Proceedings of the 7th Conference of the Internacional Organization of Citrus Virologists (pp. 186-193). Riverside: IOCV. 
Rouse, R. E. (2000). Citrus fruit quality and yield of six Valência clones on 16 rootstocks. Proceedings of the Annual Meeting of the Florida State Horticultural Society, 13, 112-114.

Salibe, A. A. (2009). Clones nucelares de citros no Estado de São Paulo. Laranja, 30(1-2), 117-136.

Salibe, A. A. (2017). Production, selection and commercial use of citrus nucellar clones in Brazil. Citrus Research \& Technology, 30(1-2), 117-136.

Saunt, J. (2000). The sweet orange. In J. Saunt (Eds.), Citrus varieties of the world (pp.13-40). Bowthorpe: Sinclair International Limited.

Tazima, Z. H., Auler, P. A. M., Neves, C. S. V. J., Yada, I. F. U., \& Leite, R. P. J. R. (2008). Comportamento de clones de laranja Valência na região norte do Paraná. Revista Brasileira de Fruticultura, 30, 970-974.
United States Department of Agriculture - USDA. (2017). Florida Citrus Statistics 2015-2016. Tallahassee: Florida Department of Agriculture and Consumer Services. Recuperado em 28 de maio de 2019, de https://www. nass.usda.gov/Statistics_by_State/Florida/Publications/ Citrus/Citrus_Statistics/2016-17/fcs1617.pdf

Recebido: Setembro 30, 2019

Aceito: Junho 23, 2020

Como citar: Stuchi, E. S., Girardi, E. A., Silva, S. R., Sempionato, O. R., Parolin, L. G., Müller, G. W., \& Donadio, L. C. (2020). Desempenho de clones pré-imunizados de laranjeiras Valência e Natal no Norte do Estado de São Paulo. Citrus Research \& Technology, 41, e1057. https://doi.org/10.4322/crt.20919 